\title{
PAPEL DE LA OXIGENOTERAPIA DE ALTO FLUJO EN LA INSUFICIENCIA RESPIRATORIA AGUDA
}

\begin{abstract}
Joan-Ramon Masclans ${ }^{1,3}$, Purificación Pérez-Terán ${ }^{2}$, Oriol Roca ${ }^{2,3}$
${ }^{1}$ Servicio de Medicina Intensiva. Hospital del Mar-Parc de Salut Mar. Institut Mar d'Investigacions mèdiques (IMIM). Barcelona. ${ }^{2}$ Servicio de Medicina Intensiva. Área General. Hospital Universitario Vall d'Hebron. Institut de Recerca Vall d'Hebron (VHIR). UAB. Barcelona. ${ }^{3}$ Ciber Enfermedades Respiratorias, Instituto de Salud Carlos III, Madrid.
\end{abstract}

Dr. Joan-Ramon Masclans

Servicio Medicina Intensiva

Parc de Salut Mar. Hospital del Mar.

Passeig Marítim, 25-29.

08003. Barcelona.

Tlfno/FAX: 932483014

jrmasclans@parcdesalutmar.cat 


\section{CONFLICTOS DE INTERESES}

Fisher \& Paykel colabora con el IMIM mediante una beca de investigación predoctoral. 


\section{PAPEL DE LA OXIGENOTERAPIA DE ALTO FLUJO EN LA INSUFICIENCIA RESPIRATORIA AGUDA}

\section{PALABRAS CLAVE}

Oxigenoterapia de alto flujo, insuficiencia respiratoria aguda.

\section{KEY WORDS}

High-flow oxygen therapy, acute respiratory failure. 


\section{RESUMEN}

La insuficiencia respiratoria aguda (IRA) supone una de las causas más frecuentes de ingreso en los servicios de medicina intensiva y la oxigenoterapia sigue constituyendo una terapéutica de primera línea en el manejo de estos pacientes. En los últimos años, la oxigenoterapia de alto flujo (OAF) ha sido descrita como una alternativa útil a la oxigenoterapia convencional en los pacientes con IRA. La OAF permite administrar un flujo de gas totalmente acondicionado hasta a $60 \mathrm{~L} / \mathrm{min}$ mediante cánulas nasales, obteniendo una rápida mejoría de los síntomas debido a diferentes mecanismos como por ejemplo una reducción de la resistencia de vía aérea superior, cambios en el volumen circulante y la generación de cierto grado de presión positiva. Además, todo ello se consigue junto con una mejor tolerancia y confort por parte del paciente. Sin embargo, la experiencia en adultos es todavía limitada y no existen guías clínicas que establezcan recomendaciones para su uso. En este artículo se pretende revisar la evidencia existente sobre el uso de OAF en pacientes adultos con IRA, así como sus posibles aplicaciones, ventajas y limitaciones. 


\section{ABSTRACT}

Acute respiratory failure (ARF) represents one of the most common causes of intensive care unit admission and oxygen therapy remains the first-line therapy in the management of these patients. In recent years, high-flow oxygen via nasal cannula (HFNC) has been described as a useful alternative to conventional oxygen therapy in patients with ARF. HFNC rapidly alleviates symptoms of ARF and improves oxygenation by several mechanisms, including dead space washout, reduction in oxygen dilution and inspiratory nasopharyngeal resistance, a moderate positive airway pressure effect that may generate alveolar recruitment and an overall greater tolerance and comfort with the interface and the heated and humidified inspired gases. However, the experience in adults is still limited and there are no clinical guidelines to establish recommendations for their use. This article aims to review the existing evidence on the use of HFNC in adults with ARF and its possible applications, advantages and limitations. 


\section{INTRODUCCIÓN}

La IRA supone una causa frecuente de ingreso en los servicios de medicina intensiva $^{1}$ y la oxigenoterapia sigue constituyendo, sin lugar a dudas, uno de los tratamientos de primera línea en su manejo². En condiciones de IRA, podemos administrar oxígeno de una manera invasiva o no invasiva. Sin embargo, siempre que sea posible, se debería tratar de evitar la instauración de un soporte ventilatorio invasivo.

Existen distintas formas de aplicación de oxigenoterapia no invasiva como por ejemplo mediante máscara nasobucal o a través de cánulas nasales. Sin embargo, la oxigenoterapia no invasiva presenta serie de factores limitantes que condicionan su eficacia y, en consecuencia, también limitan la corrección de la hipoxemia y de la clínica asociada a la IRA. Los principales factores limitantes son, por un lado, la tolerancia del sistema de aplicación y por otro la limitación del flujo de oxígeno administrado. Este, en general, se limita a 15 $\mathrm{L} / \mathrm{min}^{3}$ y normalmente se administra en condiciones no ideales de temperatura y humedad $\left(37^{\circ} \mathrm{C}\right.$ y $100 \%$ de humedad relativa). Esta limitación del flujo administrado supone que se produzca una dilución del oxigeno administrado con el aire ambiente, condicionado por el pico de flujo inspiratorio del paciente. Por tanto, a mayor pico de flujo inspiratorio, mayor dilución, con lo que disminuye la $\mathrm{F}_{1} \mathrm{O}_{2}$ real suministrada al paciente ${ }^{2,4}$. Esta situación, con poca traducción clínica en pacientes con hipoxemia leve, implica que, en pacientes con IRA grave que presentan una hipoxemia importante y que realizan picos de flujo inspiratorios mayores de $30 \mathrm{~L} / \mathrm{min}$, la corrección de la IRA pueda no ser suficiente mediante oxigenoterapia convencional. Una alternativa que solventaría estas limitaciones de la oxigenoterapia convencional es el uso de 
dispositivos de ventilación mecánica no invasiva (VMNI), si bien la principal limitación de estos dispositivos es el discomfort y la mala tolerancia por parte del paciente de sus interfaces ${ }^{5}$.

Una reciente alternativa es la oxigenoterapia de alto flujo $(\mathrm{OAF})^{6}$, que permite suministrar un flujo de gas de hasta $60 \mathrm{~L} / \mathrm{min}$ mediante unas cánulas nasales de silicona, con el gas suministrado acondicionado a nivel de temperatura y humedad ideales $\left(37^{\circ} \mathrm{C}\right.$ y $100 \%$ de humedad relativa). Hasta hace pocos años esta técnica de oxigenoterapia se había utilizado fundamentalmente en neonatos $^{7-9}$. Sin embargo, su uso en pacientes adultos ha ido incrementándose exponencialmente a lo largo de los últimos años ${ }^{10}$.

La utilización de la OAF permite una mejora en la oxigenación por una serie de mecanismos distintos, como son la disminución de la dilución del oxígeno administrado con el aire ambiente ${ }^{11,12}$, la disminución del espacio muerto ${ }^{12-14}$, el aumento del volumen circulante ${ }^{15,16}$ y la generación de presión positiva en la vía aérea $(\mathrm{CPAP})^{16-19}$. También podría producir efectos beneficiosos a nivel hemodinámico ${ }^{20}$, mejorar la capacidad para la realización de esfuerzos e incrementar el confort ${ }^{5}$ y, gracias a la humidificación activa del gas administrado, mejorar el transporte mucociliar ${ }^{21,22}$.

El objetivo del presente artículo es realizar una puesta al día de las posibles aplicaciones clínicas de la OAF, analizando los mecanismos por los que la OAF puede ser útil en pacientes adultos con IRA, en qué pacientes puede ser más útil y cómo debemos usarlo. 


\section{EQUIPAMIENTO}

Para la administración de OAF se necesitan 4 componentes: 1) interface con el paciente, 2) sistema de administración de alto flujo que permita controlar el flujo y la $\mathrm{F}_{\mathrm{i}} \mathrm{O}_{2}$ administrada, 3) sistema humidificador-calefactor y 4) tubuladuras no condensantes (Figura 1).

\section{Interface}

La interface que se utiliza son unas cánulas nasales especiales hechas de silicona y más largas que las cánulas convencionales. Existen varios tamaños, $y$, por tanto, se debe seleccionar aquél que mejor se adapte a las narinas de nuestro paciente. Además, existen adaptadores para los pacientes traqueostomizados.

\section{Controlador de Flujo y $\mathrm{F}_{i} \mathrm{O}_{2}$}

Se necesita un sistema que permita administrar gas a alto flujo (0-60 L/min) y además ajustar la $\mathrm{F}_{\mathrm{i}} \mathrm{O}_{2}$ administrada. Existen 2 sistemas distintos para lograr esto:

- Mezcla de oxígeno con aire comprimido mediante dos tomas de pared independientes conectadas con una pieza en "Y" o mediante respiradores comerciales (Dräger (Lübeck, Germany)).

- Mezcla de oxígeno con aire ambiente mediante turbina (Sistema Airvo $^{T M}$, Fisher \& Paykel Healthcare Ltd (Auckland, New Zeland)). 


\section{Humidificador-calefactor}

La clave fundamental para su uso clínico es la humidificación efectiva del gas administrado. Los sistemas comerciales más utilizados son el sistema Optiflow $^{\mathrm{TM}}$ (Fisher and Paykel Healthcare Ltd, Auckland, New Zeland) y el Vapotherm $^{\mathrm{TM}}$ Precision Flow (Vapotherm, Exeter, USA). Estos sistemas permiten la administración del gas calentado a temperatura corporal $\left(37^{\circ} \mathrm{C}\right)$ y con una humedad relativa del $100 \%$.

\section{Tubuladuras no condensantes}

Existen tubuladuras de distintas casas comerciales. Lo más importante a tener en cuenta en su elección es que estos sistemas deberían tener mecanismos para prevenir y minimizar la condensación en las tubuladuras, ya que se ha demostrado que este fenómeno favorece la aparición de infecciones ${ }^{23}$. 


\section{MECANISMOS FISIOLÓGICOS DE ACCIÓN Y EFECTOS CLÍNICOS DEL}

\section{ALTO FLUJO}

La OAF presenta unas características y mecanismos de acción que pueden ser especialmente beneficiosos en pacientes con IRA grave. A continuación revisaremos los principales mecanismos por los que se logran los efectos beneficiosos de la OAF.

\section{Mejoría de la oxigenación.}

La OAF presenta como uno de sus principales beneficios la mejoría de la hipoxemia de los pacientes con IRA. Este hecho se logra mediante varios mecanismos, siendo los principales: la menor dilución con el aire ambiente del gas suministrado durante la inspiración y una cierta presurización de la vía aérea dando lugar a un cierto efecto CPAP-like.

\section{Menor dilución}

Uno de los beneficios de la OAF es la posibilidad de suministrar un flujo suficiente para satisfacer el pico de demanda inspiratoria (PDI) del paciente, con lo que la $\mathrm{F}_{\mathrm{i}} \mathrm{O}_{2}$ suministrada se aproxima a la real que recibe el enfermo. Esto se debe a que el flujo de gas suministrado no se diluiría con aire ambiente (Figura 2). En condiciones normales, el PDI es de aproximadamente 30-40 $1 / \min ^{24}$, por lo tanto este podría ser a priori un rango de flujo recomendado cuando se empieza a utilizar la OAF. En pacientes con IRA grave, el PDI será probablemente mayor. Por este motivo, son precisamente estos pacientes donde el fenómeno de dilución del oxígeno es especialmente importante. Este hecho explicaría, al menos en parte, la mejora en la oxigenación de estos pacientes con la $\mathrm{OAF}^{25,26}$. Por otro lado, gracias a que se minimiza la dilución 
del oxígeno administrado con el aire ambiente, se consigue un mejor control de la concentración de oxígeno administrado ${ }^{27-29}$.

\section{Efecto CPAP-like}

Otro posible mecanismo de acción es la generación de una cierta presión positiva en vía aérea. Este incremento de presiones se ha demostrado a nivel de nasofaringe, de cavidad oral, a nivel de presiones esofágicas teleespiratorias y traqueales ${ }^{17-19}$

Varios estudios han demostrado que con la utilización de flujos entre 35 y 60 I/min se consiguen presiones medias espiratorias a nivel de faringe de 2-3 $\mathrm{cmH} 2 \mathrm{O}$ con la boca abierta y de $5-7 \mathrm{cmH} 2 \mathrm{O}$ con la boca cerrada ${ }^{18,19}$. Además, se ha demostrado que el uso de la OAF permite un aumento de la impedancia pulmonar al final de la espiración ${ }^{15,16}$, parámetro que se correlaciona con el volumen pulmonar. Así pues, este hecho es importante ya que significa que la mejoría en la oxigenación sería debida en parte a una mejora del reclutamiento alveolar $^{5,26,30}$, debido al menos en parte a este aumento de presiones de vía aérea.

\section{Disminución del espacio muerto}

Otro de los aspectos a tener en cuenta es que el flujo suministrado directamente a la nasofaringe, lava $\mathrm{CO}_{2}$ del receptáculo anatómico, lo que evita la reinhalación y proporciona un reservorio de gas fresco ${ }^{14,31}$. Este hecho reduce el espacio muerto anatómico e incrementa la ventilación alveolar ${ }^{12}$, dando lugar a una mejor tolerancia al ejercicio ${ }^{32}$, reducción de la disnea y una mejor oxigenación ${ }^{17}$. 
Disminución del trabajo respiratorio y del coste metabólico del acondicionamiento de gases

El uso de gas totalmente acondicionado (suministrado a temperatura y humedad ideales) ha demostrado una mejor tolerancia y confort en los pacientes con IRA ${ }^{5,33}$. Pero también permite una disminución de la frecuencia respiratoria $^{5,16,29}$ y una mejoría en la oxigenación. El acondicionamiento del gas administrado que permite la OAF afecta indirectamente en la oxigenación. La humidificación activa mejora la función mucociliar, facilita la expulsión de secreciones y disminuye la formación de atelectasias, lo que mejora la ratio ventilación-perfusión y oxigenación ${ }^{5}$. Además, la administración de gas totalmente acondicionado, permite una disminución en la resistencia de la vía aérea lo que disminuiría el trabajo respiratorio del paciente $e^{4,26}$.

\section{Efecto sobre el patrón ventilatorio y hemodinámico}

Durante la inspiración, la presión negativa limita el flujo inspiratorio debido al colapso que se produce en la nasofaringe. La OAF, al generar cierto grado de presión positiva en la vía aérea, permite contrarrestar este fenómeno ${ }^{34}$. Dado que la OAF permite administrar flujos iguales o mayores al PDI del paciente, podría minimizar la resistencia de la vía aérea superior disminuyendo el trabajo respiratorio $^{12}$. Se ha demostrado además una mejoría de la sincronía toracoabdominal con la OAF en comparación con la oxigenoterapia convencional $^{35}$.

Por otra parte, el uso de OAF da lugar a un aumento de la impedancia pulmonar y, por lo tanto, a un aumento de los volúmenes tidal ${ }^{15,16}$. Este hecho se suele acompañar de una disminución de la frecuencia respiratoria sin 
cambios en la $\mathrm{P}_{\mathrm{a}} \mathrm{CO}_{2}{ }^{5,26,30}$. Tanto este cambio en el patrón ventilatorio, que podría conllevar una a reducción del trabajo respiratorio, como la generación de un cierto grado de presión positiva intratorácica podrían generar cambios en patrón hemodinámico de los pacientes en situación de IRA ${ }^{36}$. Un estudio reciente ha analizado los efectos hemodinámicos de la OAF en pacientes con insuficiencia cardíaca estable ${ }^{20}$. Estos efectos consistían en una disminución de la precarga del ventrículo derecho (medido a través de una disminución del colapso de la vena cava inferior) lo que podría suponer una mejora hemodinámica en los pacientes con IRA secundaria a disfunción miocárdica ${ }^{20}$.

\section{Mayor confort}

De forma frecuente, el fracaso de la $\mathrm{VMNI}$ se produce por la intolerancia a las interfaces. Los pacientes pueden experimentar claustrofobia o ansiedad, pueden provocar eritema, dolor o ulceración en el puente nasal, así como sequedad y congestión nasal, irritación ocular y distensión gástrica. Además, las posibles fugas aéreas añaden discomfort y pueden interferir con el disparo y ciclado del respirador.

Toda la evidencia científica que existe al respecto, refleja que la tolerancia a la OAF es tan buena o mejor que con otros métodos de oxigenoterapia ${ }^{30}$, ya que permite un mayor confort ${ }^{5,36}$, genera menos ruido $^{37}$ y menor sequedad nasal ${ }^{5}$. Además con este sistema, se facilita la comunicación oral y permite la ingesta sin desconexiones del circuito. 


\section{PRINCIPALES INDICACIONES CLÍNICAS}

Debido a los diferentes mecanismos de acción expuestos previamente, la OAF presenta un amplio espectro de aplicaciones clínicas ${ }^{38}$. Las principales áreas donde existe evidencia para su uso se muestran a continuación. Además, en la Tabla 1 se resumen estas indicaciones analizando los principales estudios publicados y el grado de evidencia existente hasta el momento.

\section{Insuficiencia respiratoria aguda}

El estudio de Roca et al $^{5}$ fue el primero en demostrar los beneficios del uso de la OAF en pacientes con IRA. Tras sólo treinta minutos de uso de la OAF evidenciaban una mejoría significativa tanto en los parámetros clínicos como fisiológicos. Estos resultados fueron confirmados posteriormente por Sztrymf et $\mathrm{al}^{30}$ quienes confirmaron una reducción de la frecuencia respiratoria y una mejoría en la oxigenación de estos pacientes.

Además, el uso de OAF permite un mejor manejo de las secreciones respiratorias $^{30,39}$, hecho que podría ser de especial importancia en los pacientes con IRA de etiología infecciosa.

Por otra parte, el uso de la OAF podría reducir la necesidad de VMNI e incluso de ventilación mecánica invasiva (VMI) en los pacientes con IRA. Este posible beneficio de la OAF se ha estudiado en 4 estudios $^{26,29,30,40}$. En el estudio de Sztrymf et al. ${ }^{30}$ se evidenciaba un fracaso de la OAF de aproximadamente un $30 \%$, lo cual es equiparable a la tasa de fracasos de la VMNI ${ }^{30}$. El grupo de Parke $^{29}$ encontró que sólo un $10 \%$ de los pacientes con IRA moderada-grave tratados con OAF necesitaban de VMI, mientras que este porcentaje ascendía hasta un $30 \%$ en los que se usaba mascarilla nasobucal. Más recientemente, 
en una población trasplantados pulmonares con IRA, la OAF disminuía significativamente la necesidad de VMI con una reducción del riesgo absoluto del riesgo de VM del 30\%, siendo necesarios sólo tres pacientes para evitar una intubación, con potenciales efectos sobre la morbimortalidad y costeeficacia del sistema ${ }^{40}$.

Sin embargo, la cuestión principal de si el uso de OAF permite disminuir la necesidad de VMI persiste todavía sin resolver. La impresión de muchos clínicos es que efectivamente el uso de OAF evita la intubación en muchos pacientes con IRA, sin embargo no existe ningún ensayo clínico controlado que así lo demuestre. Un estudio reciente que evaluó el impacto clínico de la OAF en pacientes con IRA grave encontró una tasa de éxitos del $68 \%$, requiriendo de ventilación mecánica (ya sea invasiva o no invasiva) sólo un $32 \%$ de los pacientes $^{26}$. Todavía más llamativos son los resultados del estudio FLORALI $^{41}$, en el que se muestran los beneficios de la OAF en comparación la oxigenoterapia convencional y VMNI en términos de mortalidad y reducción de las tasas de intubación en pacientes con hipoxemia grave. En este estudio se analizaron pacientes con IRA grave tratados con OAF, oxigenoterapia convencional o VMNI. Se evidenció una menor tasa de intubación así como una reducción en la mortalidad en el subgrupo de pacientes más graves (con una relación $\mathrm{P}_{\mathrm{a}} \mathrm{O}_{2} / \mathrm{F}_{1} \mathrm{O}_{2}<200 \mathrm{mmHg}$ ) que fueron tratados con OAF.

Por otra parte, es importante conocer que existen una serie de predictores precoces (en las primeras 12 horas) del fallo de la OAF como son la persistencia de la taquipnea, la descoordinación toracoabdominal y la usencia de mejoría de la hipoxemia ${ }^{26}$. 
En resumen, en los pacientes con IRA grave el uso de OAF podría permitir: (1) Una mejoría rápida de la disnea; (2) Mejoría de la hipoxemia; (3) Mejor manejo de las secreciones respiratorias; (4) Una disminución la necesidad de ventilación mecánica.

\section{Preoxigenación en la maniobra de la intubación traqueal}

En los pacientes críticos la maniobra de intubación orotraqueal se asocia hasta con un $20 \%$ de complicaciones potencialmente graves, básicamente relacionadas con la aparición de hipoxemia en el contexto del procedimiento ${ }^{42}$. La VMNI podría utilizarse para preoxigenar al paciente que va a ser intubado. Sin embargo, ha de retirarse durante la laringoscopia y, por lo tanto, no permite la oxigenación del paciente durante el procedimiento. En cambio, el uso de OAF no interfiere con la laringoscopia y por tanto permitiría la administración de oxígeno durante el procedimiento ${ }^{14}$. Miguel-Montanes et al. ha demostrado que, con el uso de la OAF durante la preoxigenación, se reduce la prevalencia de hipoxemia grave durante la maniobra de intubación cuando se compara con el uso de una mascarilla con reservorio ${ }^{43}$. Del mismo modo, en un estudio experimental realizado recientemente se demostró que la administración de oxigeno a flujos de $10 \mathrm{~L} / \mathrm{min}$, retrasa la aparición de hipoxemia grave durante la apnea, lo que sugiere que el uso de OAF durante la intubación orotraqueal podría ser útil sobre todo en pacientes hipoxémicos ${ }^{42}$.

\section{Weaning de la ventilación mecánica}

El uso de la OAF tras retirar la VMI podría ser útil tanto para prevenir como para tratar la insuficiencia respiratoria post-extubación ${ }^{14,44}$. Se han publicado 4 estudios al respecto y todos han demostrado una mejoría de todos los 
parámetros respiratorios ${ }^{45,46}$ así como el confort si se compara con el uso de mascarilla nasobucal ${ }^{28,46}$, una disminución en la necesidad de aumento del soporte respiratorio ${ }^{44}$, además de una reducción de la tasa de reintubación (del $21 \%$ al $4 \%)^{46}$. Recientemente, el grupo de Bérard ${ }^{47}$ ha publicado un estudio de no inferioridad de OAF frente a VMNI,en concreto BiPAP, en pacientes postoperados cardíacos en riesgo de insuficiencia respiratoria, este estudio no evidencia diferencias en cuanto a fracaso del tratamiento ni mortalidad en UCI entre los dos sistemas y en cambio sí muestra que el uso de OAF se relaciona con una disminución significativa de lesiones cutáneas ya dentro de las primeras 24 horas de uso. Por otro lado, el uso de OAF en el proceso de decanulación de los pacientes traqueostomizados es otra área prometedora de desarrollo, sin embargo no existe ningún trabajo publicado en el momento actual.

\section{Oxigenoterapia durante procedimientos invasivos}

Durante la realización procedimientos invasivos como son las fibrobroncoscopias es habitual que se produzca un empeoramiento de la hipoxemia en relación a la hipoventilación que se produce y al uso de sedantes. Este empeoramiento de la hipoxemia puede ser todavía mayor si se realiza un lavado bronchoalveolar y puede persistir varias horas después de la realización de la exploración ${ }^{48}$. El uso de OAF ha demostrado efectos beneficiosos en cuanto a menor empeoramiento de la hipoxemia y confort si se compara con la oxigenoterapia convencional ${ }^{48,49}$. Recientemente, el estudio de Simon et $\mathrm{al}^{50}$. mostró una menor grado de hipoxemia durante la realización de la fibrobroncoscopia a favor de la VMNI en los pacientes con hipoxemia grave, sin embargo la tolerancia y el comfort de estos pacientes seguía siendo muy 
superior con el uso de $\mathrm{OAF}^{50}$. Del mismo modo, la utilización de OAF también debería considerarse en la realización de otros procedimientos invasivos como podrían ser las ecocardiografías transesofágicas o las endoscopias digestivas $^{14,31}$.

\section{Insuficiencia Cardíaca}

No es infrecuente encontrar pacientes con insuficiencia cardíaca aguda que, una vez estabilizados, persisten hipoxémicos y disneicos a pesar de la oxigenoterapia convencional. Los resultados de un estudio reciente sugieren que en estos pacientes la OAF mejora la disnea debido a una reducción de la frecuencia respiratoria y a una mejoría de la hipoxemia ${ }^{36}$. Por otra parte, también se ha observado que, en pacientes con insuficiencia cardíaca crónica en fase estable, la OAF daba lugar a una disminución en el colapso de vena cava inferior sin disminución el gasto cardíaco, sugiriendo que el uso de OAF provocaba una disminución de la precarga de ventrículo derecho probablemente secundaria a la generación de cierta presión positiva intratorácica y al aumento del volumen pulmonar ${ }^{20}$. Por lo tanto, la mejoría que proporciona la OAF en este tipo de pacientes podría deberse fundamentalmente tanto a la mejoría de la oxigenación como a la mejoría del patrón hemodinámico ${ }^{11,20,36}$. Sin embargo, no existe ningún estudio que compare VMNI con OAF en la fase aguda del edema pulmonar.

\section{Utilización fuera del Servicio de Medicina Intensiva}

La hipoxemia y la disnea también son unos de los síntomas más frecuentes en los pacientes ingresados en los servicios de Urgencias. Lenglet et al. ${ }^{37}$ analizaron los potenciales efectos beneficiosos y la factibilidad del uso de la 
OAF en pacientes con IRA (secundaria en su mayoría a una infección respiratoria o edema agudo de pulmón) ingresados en Urgencias comparándola con la oxigenoterapia convencional. Los resultados evidenciaron que la OAF conseguía una mejoría más rápida de la sensación disneica, oxigenación y demás parámetros respiratorios, además de presentar una mejor tolerancia y ser más confortable para los pacientes, sin que su uso presentase mayor dificultad que el uso de la oxigenoterapia convencional. Su uso en el transporte extrahospitalario queda todavía por estudiar.

\section{Cuidados paliativos}

En los pacientes en los que la VMI no está indicada, el uso de la OAF podría ser beneficioso ${ }^{15}$ ya que este dispositivo permite una disminución de la sensación disneica, mejoría de la hipoxemia y de la tos, mediante un dispositivo que presenta buena tolerancia por parte de los pacientes y que les permite al paciente comunicarse e incluso alimentarse ${ }^{37,51-53}$. De hecho, una constante en muchos de los estudio publicados hasta la fecha es la mejoría en el confort de los pacientes tratados con OAF.

\section{Exacerbación de la EPOC}

En el caso de la insuficiencia respiratoria hipercápnica, la OAF también podría ser beneficiosa en términos de reducción de la $\mathrm{PaCO}_{2}$ y mejora del $\mathrm{pH}$ probablemente en relación al aumento de presión que genera a nivel de vía aérea superior ${ }^{54}$. Sin embargo, es importante recalcar que, en estos pacientes, podría ser útil la administración de altos flujos de gas siempre y cuando

utilicemos $\mathrm{F}_{1} \mathrm{O}_{2}$ bajas para disminuir el riesgo hipercapnia. Además, estos pacientes con patología respiratoria crónica se benefician especialmente de la 
mejoría de la función mucociliar que favorece la $O A F^{54,55}$. Sin embargo, son necesarios más estudios que comparen el uso de OAF con la VMNI/CPAP en este subgrupo de pacientes. 


\section{INICIO DE LA OAF}

En la Tabla 2 se resumen los flujos de gas utilizados en los diferentes estudios.

En general, se podría utilizar un flujo inicial entre 30 y $40 \mathrm{~L} / \mathrm{min}$. En cuanto a la $\mathrm{F}_{\mathrm{i}} \mathrm{O}_{2}$, debemos administrar la necesaria para obtener la saturación arterial de oxígeno deseada. Por otra parte, es importante asegurarnos que el sistema alcanza la temperatura adecuada $\left(37^{\circ} \mathrm{C}\right)$ para la administración del gas. 


\section{WEANING DEL SISTEMA}

Es difícil establecer pautas para el destete de la OAF y su sustitución por un sistema de oxigenoterapia convencional. Sin embargo, parecería razonable primero disminuir la $\mathrm{F}_{\mathrm{l}} \mathrm{O} 2 \mathrm{y}$, posteriormente, el flujo ${ }^{6}$. Una recomendación aceptable podría ser la de mantener el flujo administrado hasta alcanzar una oxigenación correcta con una $\mathrm{F}_{1} \mathrm{O}_{2}$ menor de 0.5 . La reducción en el flujo debería ser lenta (5 I/min cada 6-8 horas). Finalmente, cuando obtengamos una oxigenación correcta con $20 \mathrm{~L} / \mathrm{min}$ o menos y con una $\mathrm{F}_{\mathrm{l}} \mathrm{O}_{2}$ menor de 0.5 , podríamos plantearnos la sustitución de la OAF por un sistema de oxigenoterapia convencional ${ }^{56}$. No obstante, en algunos pacientes es necesario el uso de OAF de forma intermitente durante la fase de destete. 


\section{CONTRAINDICACIONES Y COMPLICACIONES}

No se han descrito efectos adversos importantes en relación a la utilización de la OAF. Los sistemas de humidificación activa permiten administrar gas totalmente acondicionado y, por lo tanto, los efectos secundarios a nivel de las estructuras nasofaríngeas se minimizan enormemente. En los pacientes con EPOC, la utilización de altas concentraciones de oxígeno puede producir acidosis respiratoria debido a la reducción de la frecuencia respiratoria y las alteraciones de la ventilación-perfusión ${ }^{57}$. Existen varios sistemas de alto flujo disponibles. El ideal es aquél que resulte más confortable para el paciente y que tenga el mejor sistema de humidificación y calentamiento con tubuladuras no condensantes. Estos sistemas deberían tener mecanismos para prevenir y minimizar la condensación en las tubuladuras, ya que este fenómeno favorece la aparición de infecciones, como por ejemplo brote de colonización o infección por Ralstonia reportado por la FDA con el sistema de Vapotherm ${ }^{23}$, ya controlado en la actualidad. 


\section{CONCLUSIONES}

En resumen, la OAF es una nueva opción de oxigenoterapia que, mediante el calentamiento y humidificación permite la administración de gas totalmente acondicionado a muy altos flujos, mejorando la oxigenación y el confort de los pacientes y minimizando los efectos adversos sobre las estructuras nasofaríngeas. Con la evidencia actual, la OAF es una opción terapéutica atractiva y útil en los pacientes con IRA ya que permite una mejoría de la oxigenación, disminución del trabajo respiratorio y mayor confort de los pacientes. Sin embargo, son necesarios más estudios que determinen su posible impacto en términos de morbimortalidad y coste-efectividad. 


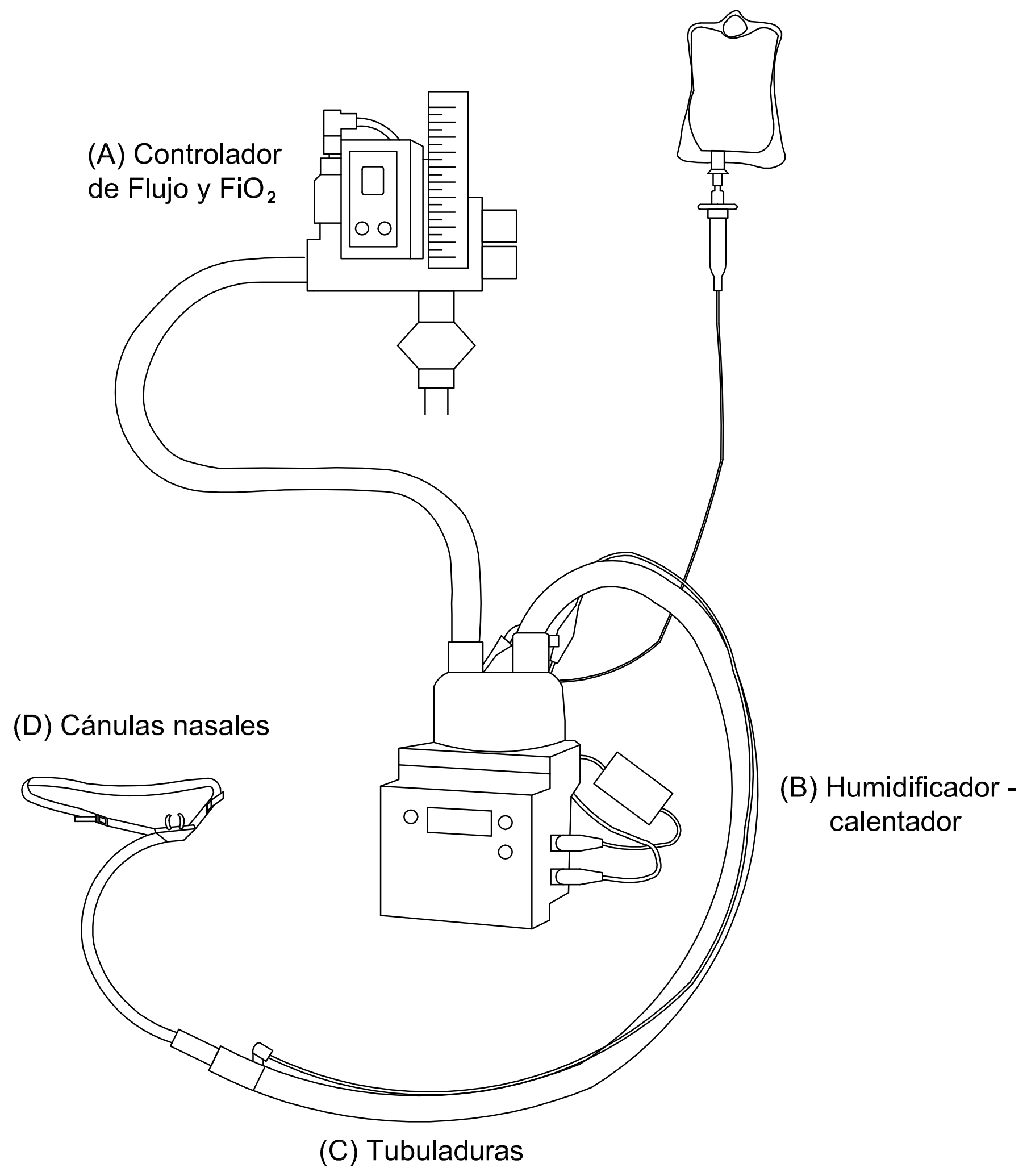


a) Cánulas nasales 4 lpm

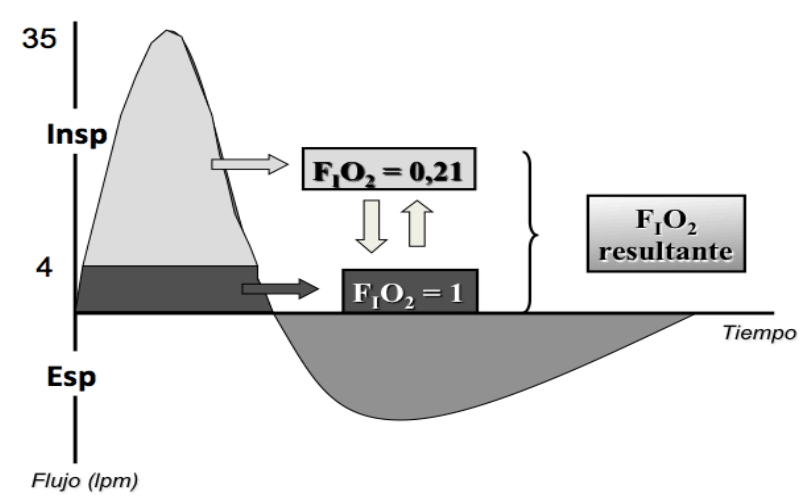

b) Cánulas nasales $35 \mathrm{lpm}$

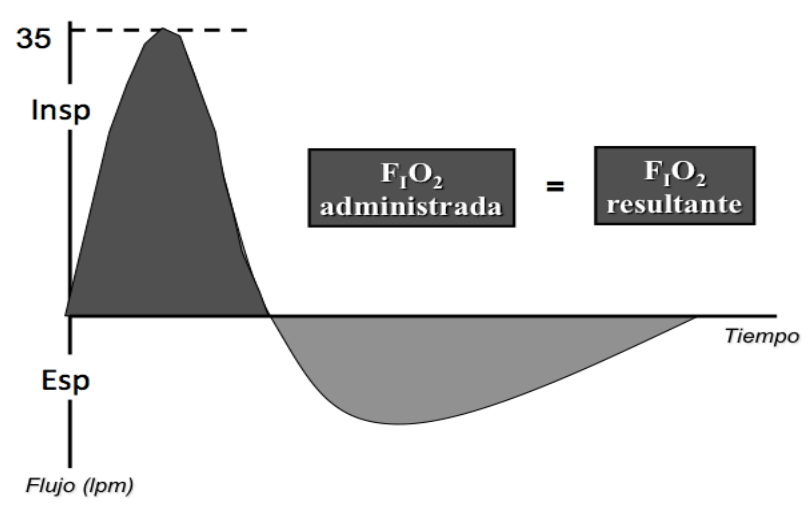

